\title{
Isolating attentional systems: A cognitive-anatomical analysis
}

\author{
MICHAEL I. POSNER \\ University of Oregon, Eugene, Oregon
}

\begin{abstract}
ALBRECHT WERNER INHOFF and FRANCES J. FRIEDRICH
Cognitive Neuropsychology Laboratory, Good Samaritan Hospital, Portland, Oregon
\end{abstract}

and

\author{
ASHER COHEN \\ University of Oregon, Eugene, Oregon
}

\begin{abstract}
Recently our knowledge of the mechanisms of visual-spatial attention has improved because of studies employing single cell recording with alert monkeys and others using performance analysis of neurological patients. These studies suggest that a complex neural network that includes parts of the posterior parietal lobe and midbrain is involved in covert shifts of visual attention. Is this system an isolated visual attentional module or is it part of a more general attentional system? Our studies employ the dual-task technique to determine whether covert visual orienting can take place while a person's attention is engaged in a language processing task. We find clear evidence of interference between the two tasks, which suggests some common operations. However, the results also indicate that whatever is common to the two tasks does not have the same anatomical location as that of visual-spatial attention.
\end{abstract}

A fundamental problem in the study of attention is to understand how the unity of conscious experience is related to the many levels of selectivity involved in processing external events. The amount of information of which we are aware at any moment seems remarkably limited, yet it is often efficiently selected from a vast array of input. We are generally unaware of the details of the selection, but without it our subjective experience could not remain unified.

In recent years a more detailed anatomical and physiological analysis of attention has developed within the domain of selection of visual-spatial information (Mountcastle, 1978; Posner, 1980; Wurtz, Goldberg, \& Robinson, 1980). This work involves studies of alert monkeys and of normal and brain injured patients orienting to visual events. Since no overt changes (e.g., eye movements) need occur for there to be evidence of selection at the attended location, it is possible that mechanisms revealed by these studies may serve as a model for understanding attention in general.

At the level of computations, one can view a shift of visual attention as involving three more elementary operations isolated from chronometric studies. The first operation is disengaging from the current focus of attention. It is a well established principle that the difficulty of pro-

This research was supported in part by NIMH Grant R01-3853-02 and in part by the Office of Naval Research under Contract Nos. N 0014 83-K-0601 and N 0014-86-K-0289. Requests for reprints should be sent to Michael I. Posner, Department of Neurology, Box 8111, Washington University School of Medicine, 660 S. Euclid, St. Louis, MO 63110. cessing influences the amount of time necessary to switch or disengage from that task (LaBerge, 1973). This principle is responsible for much of the use of secondary tasks to measure attention demands (see Kerr, 1973, for a review).

The second operation involves a movement of attention from its current focus to a new location. There is some reason to believe that this movement is analog in the sense of passing through intermediate locations (Shulman, Remington, \& McLean, 1979; Tsal, 1983; Ullman, 1984), but this is by no means settled (Hughes \& Zimba, 1985; Remington \& Pierce, 1984). The move operation could be similar to the operation involved in mental rotation and image scanning (Kosslyn, 1980; Pinker, 1980; Shepard, 1978).

Finally, the subject must engage the new target. The engage operation is likely to differ according to the task required. Some processing (e.g., the registration of features or the looking up of highly familiar responses) may take place without engaging attention (Marcel, 1983; Treisman \& Gelade, 1980). However, it appears that attention must be at the target for an arbitrary speeded response of maximum efficiency to occur. Thus faster responses and higher $d^{\prime}$ 's are reported for events that occur at locations to which attention has been cued (Bashinski \& Bachrach, 1980; Downing \& Pinker, 1985; Posner, 1980).

Each of these operations appears to be affected by a different form of brain injury. Damage to the parietal lobe can produce a severe deficit in the ability to disengage attention from a visual location, without any loss in efficiency of the move or engage operations (Posner, Walker, Friedrich, \& Rafal, 1984). Our results show only small 
differences in reaction time (RT) to targets in either visual field once attention has been brought to that location by a cue (valid trials). Moreover, the improvement in RT to a target following a valid cue is about the same for targets in the contralateral and ipsilateral fields. If this improvement is due to a shift of attention to the cued side, it follows that the ability to move attention must not differ between the two fields. However, if attention is first cued to fixation (or to the visual field ipsilateral to the lesion), there is a massive increase in RT (of several hundred msec) for targets that occur on the side opposite the lesion. Thus, once attention is engaged at fixation (or at another place), the patient seems to have great trouble in disengaging it to deal with targets contralateral to the focus of attention (Posner, Cohen, \& Rafal, 1982; Posner, Walker, Friedrich, \& Rafal, 1984). We call this result the extinction-like reaction time pattern because it resembles what neurologists have called extinction of a contralateral event when it is presented simultaneously with an event on the side of the lesion.

This pattern contrasts with what we have found when there is damage to the midbrain. In progressive supranuclear palsy, patients lose the ability to make voluntary eye movements (saccades). This usually occurs first for vertical eye movements and later for horizontal movements (Posner, Choate, Rafal, \& Vaughan, 1985; Posner et al., 1982). We have found that these patients are slower in shifting attention in the vertical direction than in the horizontal (Posner et al., 1985; Posner et al., 1982). For these patients, unlike for normals or other control populations, the advantage of the cued side over the uncued side emerges later when the cue requires a vertical shift of attention than when it requires a horizontal shift. The pattern is quite different for parietal patients, who show a specific deficit to contralateral targets only when they follow invalid cues. The deficit for midbrain patients occurs even for a target at a cued location, as though such patients have difficulty in moving attention either to a cue or to the target. This pattern is consistent with a deficit in the move operation because all shifts of attention in the vertical direction are affected. It is as though the patient simply has increased difficulty in shifting attention in the vertical direction.

These findings fit well with the single-cell recording data from monkeys. These data demonstrate that the parietal lobe contains cells that show enhanced responses to stimuli in their receptive fields when the animal is trained to attend to that location while maintaining fixation at another place (Wurtz et al., 1980).

Cells in the superior colliculus appear to be more closely related to attention when actual eye movements are involved (Wurtz et al., 1980). The human data also suggest the close relation of midbrain damage to eye movements. As described above, damage that affects the ability to move the eyes overtly toward a stimulus also retards the rate of the covert move operation toward that stimulus. In addition, lesions of the midbrain may increase the likelihood of attention's returning to a visual location that has recently been examined either by fixation or covertly
(Posner et al., 1985). A bias against such return movements would have obvious importance in favoring novel information during visual scanning.

Thus we can now define one form of visual-spatial attention in terms of relatively precise cognitive operations and also say something about the anatomical locus of these operations. In this paper we ask whether visual-spatial attention shares cognitive operations with other attention senses. Specifically, does attending to a language task interfere with the operations involved in orienting visualspatial attention, or are these operations independent of the language task? If visual-spatial attention and language share operations, we can use the results from our patients to attempt to establish whether these shared operations involve the parietal lobe.

\section{EXPERIMENT 1}

Our strategy was to assess the performance of patients with known deficits due to parietal lesions and that of groups of normal controls. The primary task was the visual-spatial attention orienting task studied previously. Each trial began with a visual cue that drew the person's attention to a location in space. To assess the effectiveness of the cue, we required the person to respond as rapidly as possible to targets that occurred at the cued location $80 \%$ of the time (valid trials) and at an uncued location $20 \%$ of the time (invalid trials). A single key was pressed irrespective of target location. The advantage of the cued over the uncued location in RT has been confirmed many times (Hughes \& Zimba, 1985; Jonides, 1981; Lansman, Farr, \& Hunt, 1984; Posner, 1980) and has often been attributed to the covert shift of attention to the cued location.

To study the issue of whether visual-spatial attention is a separate module, we had the subjects perform a visualspatial orienting task either by itself or combined with one of two secondary tasks. The secondary tasks involved language and were chosen in an effort to ensure the use of separate input and output paths and quite different cognitive operations from those used in visual-spatial orienting. We then examined the ability of the patients and the normals to time-share the primary and secondary tasks. If visual-spatial attention is a separate module, we would expect a general increase in RT due to interference with output or reliance on some very general common resource. However, the advantage of a shift of attention to the cued location would be expected to remain, since, if visual-spatial attention is a separate module, it could operate to shift attention even when the subject was engaged in performing the secondary task. If the secondary task shares some attentional mechanisms with visual-spatial attention, we would expect to find interference with the covert shift of attention (e.g., invalid - valid RTs) as well as an overall increase in RT. There is already some evidence that dual tasks influence the ability to shift attention toward a cue. In one study researchers found that cuing the modality of a probe (visual vs. auditory) produced significantly faster RTs for the cued modality (Lansman 
et al., 1984), but these cuing effects were reduced or eliminated when performed during a dual task. In another study, when counting backward, the subjects were affected less by spatial cues during a dual task than during a spatial orienting task alone (Posner, Cohen, Choate, Hockey, \& Maylor, 1984). It seems likely, then, that dual-task conditions may serve to reduce the effectiveness of the cues.

If attending to the language task reduces the validity effect (invalid - valid RTs), we can conclude that the spatial orienting and language tasks share a common attentional system. The system must be common because the act of processing language interferes with the validity effect; it must be attentional because, according to theory, the validity effect involves a shift of attention.

Our use of parietal patients also makes it possible to say something about the anatomy of the interference. When parietal patients attend to a cue in the good field, or at fixation, they show a specific interference in responding to targets on the side of space contralateral to the lesion. Many of these patients show no difference between ipsilateral and contralateral targets in uncued trials or in trials in which the cue is at the target location. We have interpreted this result as showing that the problem parietal patients have with contralateral targets is due to a specific deficit in their ability to disengage attention from the cue (Posner, Walker, Friedrich, \& Rafal, 1984). If attending to the language task involves the same system as does attention to a spatial location, we would expect to observe a similar specific disadvantage for a contralateral target when the patient is engaged in processing the language task. The idea is quite straightforward. When a parietal patient attends to a visual cue, we find a specific disadvantage in detecting contralateral targets, as compared with ipsilateral targets. This appears to be a specific sign of parietal damage. If attending to language uses the same system, parietal patients should show a specific disadvantage in responding to contralateral targets.

If we find that doing the language task reduces the validity effect, we can argue that the patients are attending to language. We can then ask whether attending to language produces a specific disadvantage for contralateral targets. If this occurs, then attending to the language task would be thought to use the same parietal attentional system that we have found to be involved in visual-spatial attention. If attending to language produces no specific disadvantage for contralateral targets, we can conclude that the attention system common to language and spatial orienting is quite different from that used by spatial orienting alone. The common system would not be the parietal system we have identified with visual-spatial attention.

We thus have two indices of the separability of the primary and secondary tasks. The first has to do with whether the secondary task reduces the advantage of valid over invalid RTs when the primary task is performed alone. This index allows us to determine whether the two tasks involve the same or different cognitive operations. The second index tells us whether the secondary task produces a greater specific deficit for invalid targets contra- lateral to the lesion. This tells us whether attending to the secondary task involves the same anatomical system as does attending to a visual location.

\section{Method}

Subjects/patients. Nine parietal patients were studied, using two different secondary tasks. The first, which involved counting backward from a three-digit number, was used with only 5 patients. The second, which involved monitoring a series of auditory words and counting the number of phonemes, was used with all 9 patients. The 9 patients used in this study all had unilateral lesions of the parietal lobe confirmed by computerized tomography (CT) scan: 4 patients had lesions on the left side, and 5 had lesions on the right side. All of the lesions resulted from stroke. Two of the patients with right-hemisphere lesions were 5 or more years poststroke. All of the right-hemisphere patients had aspects of neglect and extinction at the time of the stroke. However, only one of them had clear problems at the time of testing. This patient, J.C., had a field cut that affected visual processing for stimuli more than $5^{\circ}$ from fixation. Within the field cut he showed evidence of neglect. The lefthemisphere patients did not appear to have clear neglect problems. However, 3 of them had some form of aphasia as a result of the lesion. Three of 9 patients had damage that extended beyond the parietal lobe into the frontal area; 2 of the 9 patients had temporal damage in addition to the frontal damage. Further data on the lesions of 3 of the patients (J.C., W.K., and E.A.) are presented in Table 1 of Posner, Walker, Friedrich, and Rafal (1984). In that study of 13 patients, we found that the deficit in the disengage operation correlated with the extent of parietal lobe removed, and the best correlation was with the extent of superior parietal lobe removed. Such factors as lesion size and posterior or frontal extent of the lesion did not correlate with the disengage deficit. Because several of these patients were used in the earlier study and because we had only 9 patients for this study, we did not attempt to reexamine these correlations.

Control subjects. Sixteen subjects without documented neurological disorders served as controls for the parietal patients. Eight of the control subjects were in the 19 to 35 age group and were recruited from the staff of Good Samaritan Hospital and Medical Center or Portland State University; the other 8 control subjects were from 60 to 75 years old.

Tasks. In the single-task condition, subjects were required only to detect the visual target and to depress a single key with the index finger as quickly as possible. The basic experimental paradigm was similar to that used in Posner, Walker, Friedrich, and Rafal (1984). Subjects faced a cathode-ray tube (CRT) $80 \mathrm{~cm}$ from the eyes. They were instructed to maintain fixation on a central box. Two peripheral boxes were present approximately $8^{\circ}$ to the left and right of fixation.

Two types of single-task blocks were used. In cued blocks, one of the two peripheral boxes was brightened for $300 \mathrm{msec}$ by doubling the number of illuminated points that formed the box. After an interval of 100,500 , or $1,000 \mathrm{msec}$, the onset of the cue was followed by a bright asterisk that filled the box. The target asterisk was presented on the cued side $80 \%$ of the time (valid trials) and $20 \%$ of the time on the uncued side (invalid trials). In uncued blocks, the cue was omitted and only the target occurred, $1,100,1,600$, or $2,000 \mathrm{msec}$ after the previous response.

In the dual-task condition, one of two secondary tasks was added to the primary task. One secondary task involved phoneme detection. The subjects were required to monitor for the phoneme $p$ in a list of words. Specifically, the subjects were played a tape with 30 lists of 20 words each. These lists were spoken by a native speaker at a word presentation rate of approximately one word per $2 \mathrm{sec}$. Only nouns were used. In each list one to seven words began with the phoneme $p$. Immediately after the last word of a list was 
pronounced, the command "stop" was given to indicate that the visual-detection task was to be interrupted and that the last item of a list of words had been presented. After the stop command, the patient was asked how many nouns on the presented list of words had begun with the letter $p$. The question was followed by a silent interval of approximately $3 \mathrm{sec}$ within which the subject was required to report the number of words that started with the phoneme $p$. After this, a "ready" command was given to indicate that the visual-detection task was to be continued and that a new list of words was to be presented.

The backward-counting task was similar. Each block of trials was initiated by a three-digit number from which the patient counted backward by ones. In the dual-task blocks, orienting trials were conducted during the counting process. After 15 to 20 trials, the subjects rested before a new number was given.

Performance on the phoneme-monitoring task alone was measured for 5 of the patients.

Procedure. Each subject was tested under all of the conditions in a single session. At the start of the session, each was introduced to the phoneme or backward-counting task. Each then received either three blocks of no-cue trials followed by three blocks of cued trials or the reverse (the order was counterbalanced across subjects). Each block consisted of 100 trials if no cues were involved, or 300 trials for cued blocks. Within each set of three blocks an ABA design was used, so that visual orienting alone came both before and after the dual-task block.

The backward-counting task was run on 5 patients prior to the phoneme-monitoring task. This was done in a single session, and only cued trial blocks were used.

\section{Results}

The main results of the experiment are described in terms of RTs for the spatial-attention task when performed alone and in conjunction with the monitoring task. The median RTs for each condition were calculated for each subject. All RTs less than $100 \mathrm{msec}$ or greater than $3,000 \mathrm{msec}$ were excluded (these represented less than $1 \%$ of the trials). Overall results were quite similar for the trials in which there was a 100 -msec delay following the cue and for the trials in which the delay was longer. Since $2 / 3$ of the trials were run at the 100 -msec interval and these trials were free of any eye movements, they seemed most appropriate for discussion. Although the analyses of variance (ANOVAs) reported include all delay intervals, we discuss the longer intervals only in those cases where interval interacted with other effects.

The overall data from the primary task that had phoneme monitoring as the secondary task were cast into two ANOVAs. One involved the patient groups and had side of lesion as the between-subjects condition and attention (focused vs. divided), cue (valid, invalid, no cue), visual field (ipsilateral vs. contralateral to lesion), and interval (short vs. delayed) as the within-subject variables. The second analysis involved only the control subjects and had age as the between-subjects variable ( 8 old and 8 young), with the same set of within-subject variables except that visual field was left versus right. The data from the 5 subjects who counted backward were also summarized, but were not analyzed statistically.

Figure 1 shows both valid and invalid trials from cued trial blocks for each of the four subject groups. The results confirm the findings of numerous experiments (Hughes
\& Zimba, 1985; Jonides, 1981; Posner, 1980) that showed advantages for the cued side over the uncued side in all conditions. The younger normal subjects in this experiment showed the smallest advantage of the cued side over the uncued side $[F(1,7)=5.7, p<.05]$. This was clearest at the short interval $(36 \mathrm{msec})[F(1,7)=12.1$, $p<.01$, but there was no significant interaction with interval. The older normal subjects also showed a significant advantage for the cued side $[F(1,7)=9.4, p<.01]$, which was largest ( $98 \mathrm{msec}$ ) at the 500 -msec interval, but once again there was no significant interaction with interval. The patients also showed a highly significant effect of validity $[F(1,8)=15.2, p<.01]$.

In each group, cued trials showed an advantage over noncued trials. There was no overall interaction with delay interval. In previous studies, where more intervals were used, the advantage of the cued side over the uncued side, attributed to the orienting of attention toward the cue, grew during the first $150 \mathrm{msec}$ following the cue. Possibly because so few intervals were used in the current study, we found no growth of the validity effect (advantage of cued over noncued side) with interval. The presence of a constant validity effect over intervals indicates that the effect could not be due to purely sensory influences caused by the cue, because the effect was present long after the cue went off. Since the validity effects were fully present at a 100 -msec interval, there seems to be little likelihood that overt eye shifts were mediating this effect. Moreover, in previous work it was demonstrated explicitly that the eyes remained fixed under conditions similar to those used in this experiment (Posner, 1980).

The data also confirm previous findings with parietal patients (Posner, Walker, Friedrich, \& Rafal, 1984). Both left- and right-sided patients showed a markedly greater advantage of valid over invalid trials when the target was contralateral to the lesion than when it was ipsilateral [cue $\times$ side interaction; $F(2,14)=9.3, p<.01$ ]. This has been previously reported (Posner, Walker, Friedrich, \& Rafal, 1984) and called the extinction-like reaction time pattern, since it is similar to the clinical phenomenon in which patients miss contralateral signals when they occur simultaneously with ipsilateral signals (extinction). Left- and right-sided patients both show normal validity effects on the ipsilateral side (about $50 \mathrm{msec}$ ) but markedly larger effects on the contralateral side (200 msec).

In the dual-task performance, 5 of the patients were run on the phoneme-monitoring task alone as well as with the visual task. The mean percentage of 20 trial blocks in which their report of the number of phonemes detected was correct was $68 \%$ when performed alone and $36 \%$ when combined with the visual task. The 4 patients for whom no separate blocks of phoneme monitoring alone were collected had a mean detection rate of $70 \%$ in the dual-task blocks.

Figure 2 shows valid and invalid RTs for both divided and focused conditions for the two secondary tasks. The 


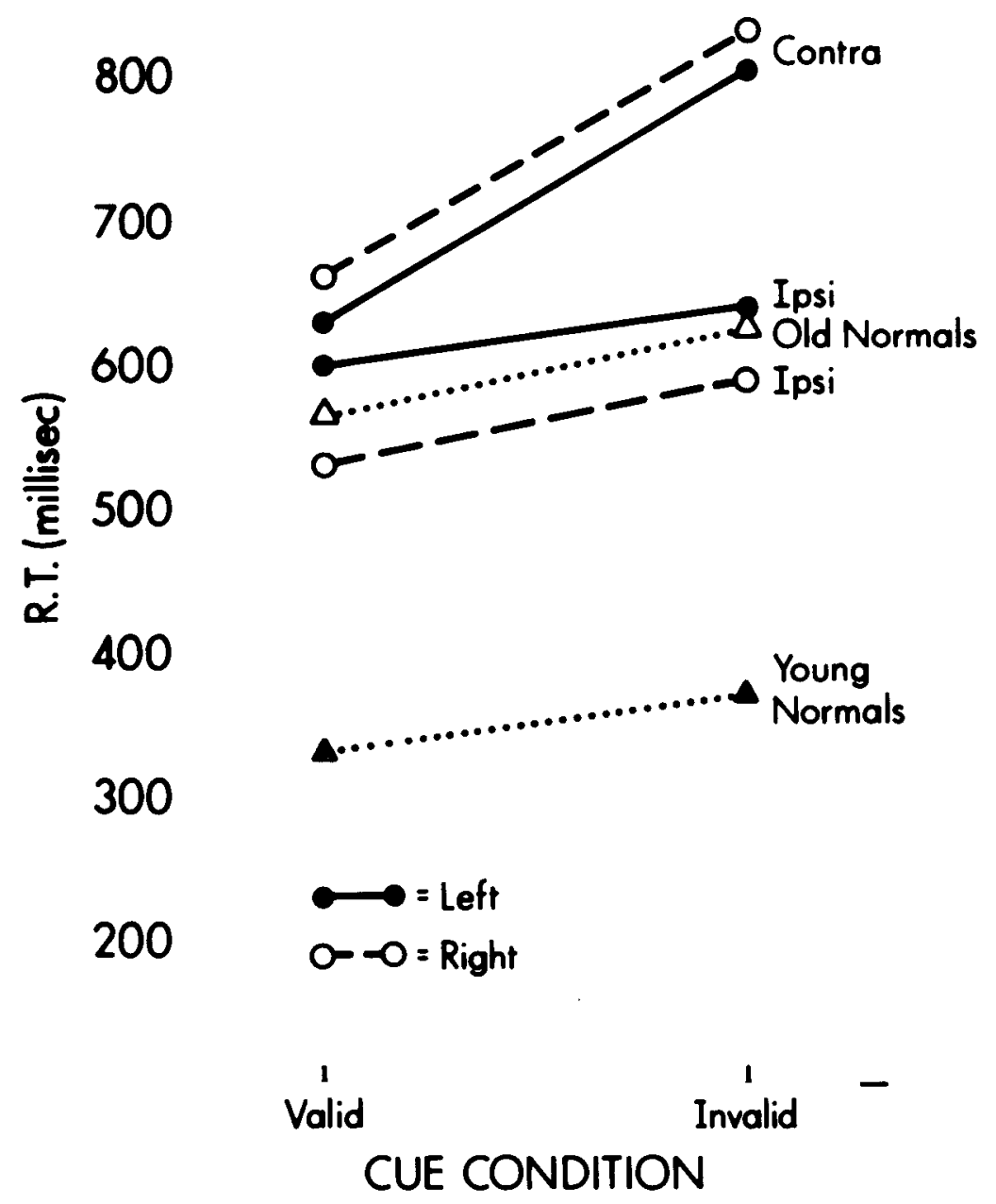

Figure 1. Mean RTs as a function of cue condition (valid vs. invalid) in the single task blocks of Experiment 1. Data are for young and old normal groups and for patients with right and with left parietal lesions. For the patient groups, the data are separated for targets on the side of the lesion (ipsilateral) and on the opposite side (contralateral).

top two lines are for the phoneme-monitoring task. It is clear that dividing attention had a powerful main effect on the spatial task RTs $[F(1,7)=10.5, p<.01]$. Moreover, dividing attention clearly eliminated the validity effect. Valid and invalid RTs were essentially identical under divided-attention (phoneme) conditions. An identical effect was found for the counting task (lower two lines). Under divided-attention conditions, there was no difference between valid and invalid RTs. Only 5 patients were run on the counting task, so no statistical analysis was done.

Figure 2 gives data for the 100 -msec delay condition for 9 patients studied in the phoneme-monitoring task. Phoneme monitoring was, however, the only place in which interval interacted significantly. There was a strong attention $x$ cue $x$ interval interaction (shown in Figure 3).
Although dividing attention abolished the validity effect at 100 msec (see Figure 2), the effect was clearly present by $500 \mathrm{msec}$ (see Figure 3 ). Thus divided attention delayed the ability of the patient to shift attention to the cue.

Normal subjects showed similar effects when attention was divided under some circumstances. Figure 4 shows the results for old and young normals in the phonememonitoring task (left panel) and for young normals in a previously reported study of counting backward by threes (Posner, Cohen, Choate, Hockey, \& Maylor, 1984). In all cases, attention affected primary task performance. However, for phoneme monitoring, divided attention clearly had no effect on the size of the difference between valid and invalid trials. When we examine the backwardcounting task (Figure 4, right panel) reported by Posner, Cohen, Choate, Hockey, and Maylor (1984), we find that 


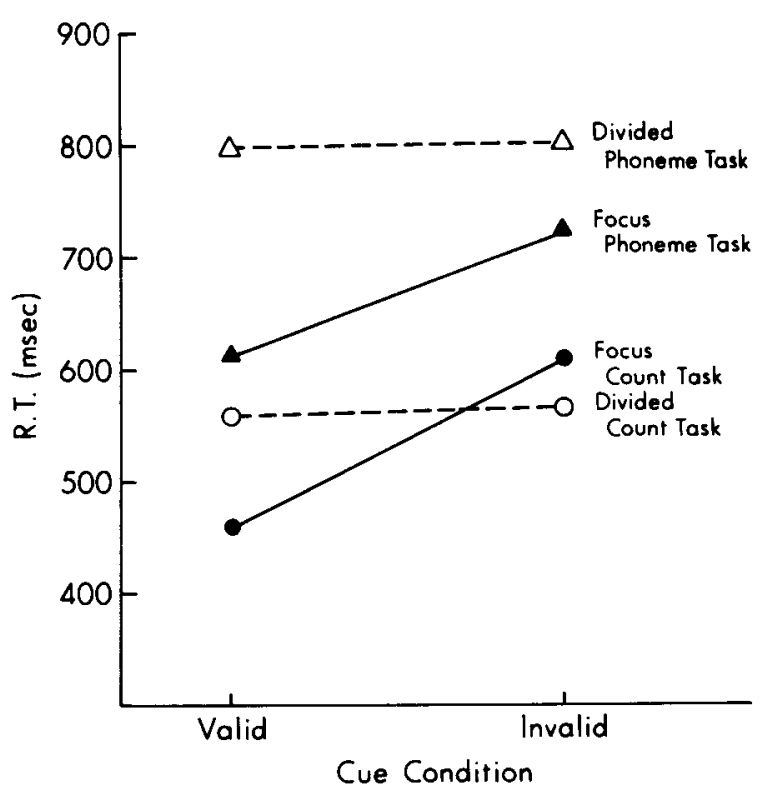

Figure 2. Mean RTs for valid and invalid trials for a spatialattention task when performed alone (focus) and when done with two types of secondary tasks: monitoring for phonemes and counting backward.

divided attention had a much greater effect on raw RTs and its interaction with validity was of the same type as found in patients. Maylor (1985) examined the dual task of backward counting and visual orienting. She found that early in practice, counting backward eliminated the facilitation due to the cue; later in practice, however, the cue was effective. In another experiment in the same study, she showed that preparation of a saccadic eye movement eliminated the facilitation effect, although pursuit movements did not. These results seem to support the view that when the secondary task involves sufficient difficulty, there is interference with orienting toward the cue.

The results illustrated in Figures 2 and 4 show that under appropriate conditions, divided attention can delay the ability of the cue to draw attention sufficiently, so that neither normals nor patients show a validity effect at $100 \mathrm{msec}$. This suggests that the spatial orienting system must share some operations with the two secondary tasks, causing a delay in orienting when they are sufficiently difficult.

One might argue that patients use the cue normally under divided-attention conditions, but that no effect is found because the language task delays the keypress. This view would regard the cue effects as being lost because the delayed RT allows the subject to shift attention from cue to target without this attention shift's showing in RT. This view, however, cannot explain the presence of a validity effect in the longer delay trials shown in Figure 3. In this delayed condition RTs were longer due to the secondary task, but now a validity effect is clearly present. If the secondary task reduced the validity effect by delaying output in the 100-msec trials, one would expect a similar ef- fect at longer intervals, since the overall delay in RT due to the dual task is still present. Instead, it appears that the longer intervals provided a differential advantage on valid trials, as one would predict if the secondary task retarded the ability to use the cue.

Why should patients not orient to the cue at the short intervals in the dual-task blocks? Clearly this must be due to the fact that they are engaged in processing the language task. If orienting to the secondary task uses the same parietal system as does visual-spatial orienting, the patients should have specific problems with invalid contralateral targets. Prediction of an extinction-like RT pattern at 100 msec follows both from the view that the patient has oriented to the cue but cannot respond because of the secondary task, and from the view that visual orienting has not taken place because the language task is engaging attention and uses the same parietal mechanism that is used for visual-spatial attention.

Table 1 shows the RTs for focused- and dividedattention conditions at 100 msec for valid and invalid targets in the ipsilateral and contralateral visual fields. Divided attention elevated RTs in all conditions except that of the contralateral invalid cue. According to our earlier theory, RTs in the contralateral invalid cue condition are inflated because damage to the parietal lobe interferes with disengaging from the visual cue when

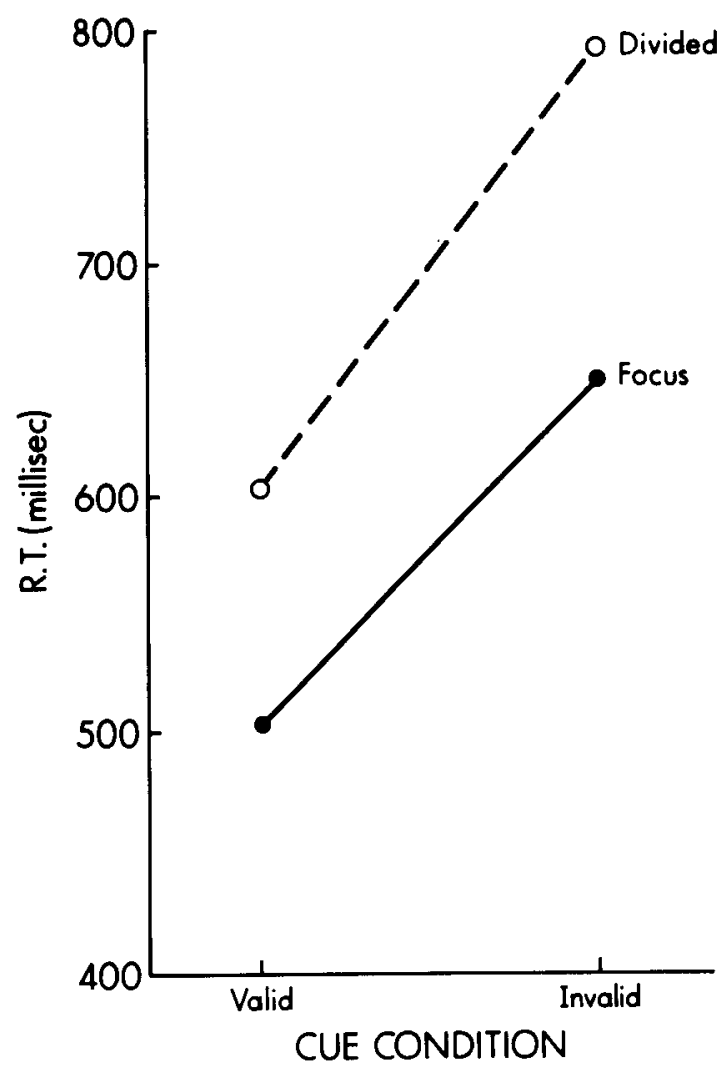

Figure 3. Mean RTs for patient groups with long delay trials (500 or 1,000 msec between cue and target) for both focused and divided blocks as a function of cue validity. 


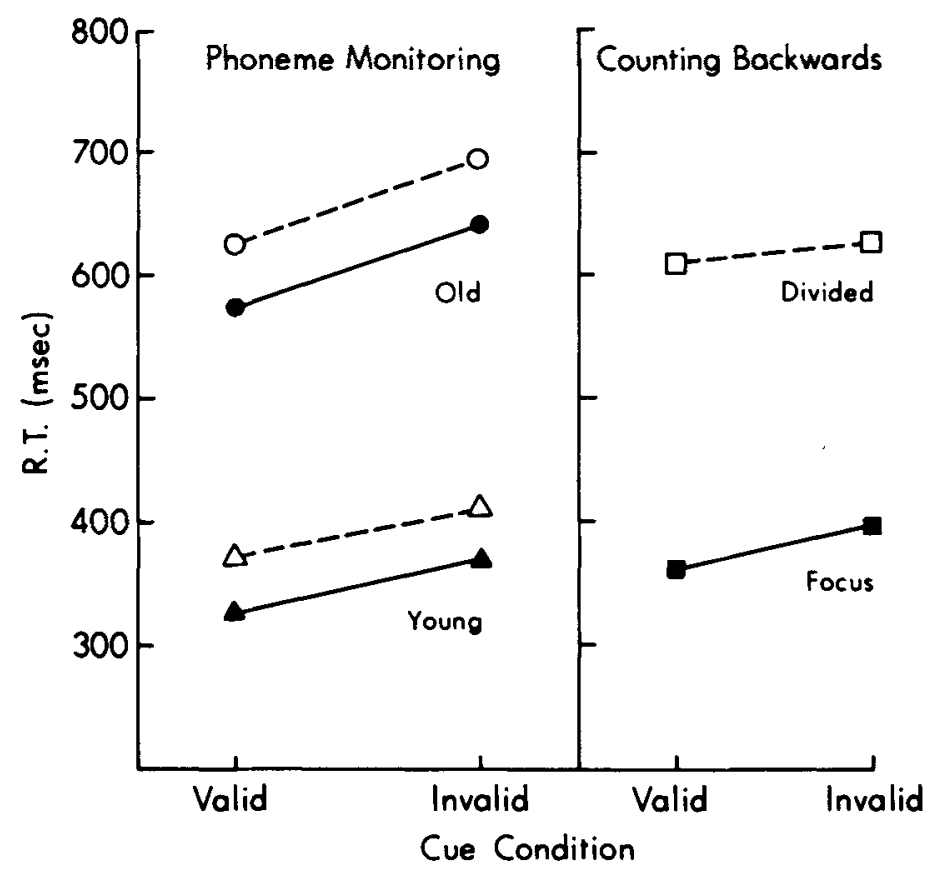

Figure 4. Mean RTs for young and old normals for spatial attention alone (solid lines) and dual task (dashed lines). Left panel involves phoneme monitoring as the secondary task. Right panel refers to data from Posner, Cohen, Choate, Hockey, and Maylor (1984) for counting backward by 3 as the secondary task.

contralateral targets are presented. If the language task prevents engaging the cue, it should also reduce the specific disadvantage for contralateral targets, provided that specific deficit does not also occur when the subject is engaged in a language task.

Divided attention eliminated the extinction-like RT pattern (contra visual field-ipsi visual field) for invalidly cued trials. This result is confirmed by a significant tripleorder interaction between validity, attention, and visual field $[F(2,14)=3.5, p<.01]$. The interaction for the 100 -msec delay interval is shown in Figure 5. The figure shows that under focused-attention conditions, RTs were greatly lengthened for contralateral invalid trials (extinction-like RT pattern), but there is no evidence of this under divided-attention conditions.

\section{Discussion}

When the patient is engaged in a visual task, there is a specific deficit in the ability to disengage to handle a contralateral target. This result, which shows up clearly under the focused condition in Figure 5, confirms our previous work. This extinction-like RT pattern is a distinctive sign of parietal lesions. However, when the patient's attention is drawn to the language task (the dividedattention condition), the increase in RT to contralateral invalid trials goes away. Thus we conclude that engaging in the language task does not involve the parietal system that we have described for visual-spatial attention.
Table 2 shows the results obtained in the no-cue condition. First, the no-cue blocks generally gave faster RTs than did the valid trials for both normals and left-sided patients. Why should this be if, as we have argued, the advantage of valid over invalid trials is due to the presence of attention? Surely valid trials should be better than those without cues, particularly because cued trials allow for increased alerting as well as for the advantages of selective attention to the cued location.

At first we were very puzzled by these results; however, we have come to view them in light of the "emergent properties" argument (Duncan, 1980a). In a simple RT task, subjects often adopt a criterion of responding to any energy change. This works so long as there are no events to which they must inhibit a response. However, in the cued paradigm the subjects must withhold a response to the cue. Thus subjects could raise their response criteria for blocks in which there are cues over those in which

Table 1

Reaction Time as a Function of Validity and Visual Field

\begin{tabular}{|c|c|c|c|c|c|c|c|c|}
\hline & \multicolumn{4}{|c|}{ Focused } & \multicolumn{4}{|c|}{ Divided } \\
\hline & \multicolumn{2}{|c|}{ Valid } & \multicolumn{2}{|c|}{ Invalid } & \multicolumn{2}{|c|}{ Valid } & \multicolumn{2}{|c|}{ Invalid } \\
\hline & Contra & Ipsi & Contra & Ipsi & Contra & Ipsi & $\overline{\text { Contra }}$ & $\overline{\text { Ipsi }}$ \\
\hline & 642 & 570 & 778 & 622 & 748 & 680 & 778 & 715 \\
\hline Contra-Ipsi & 72 & & 156 & & 68 & & 63 & \\
\hline
\end{tabular}

Note-All data from 100-msec cue-to-target interval. 


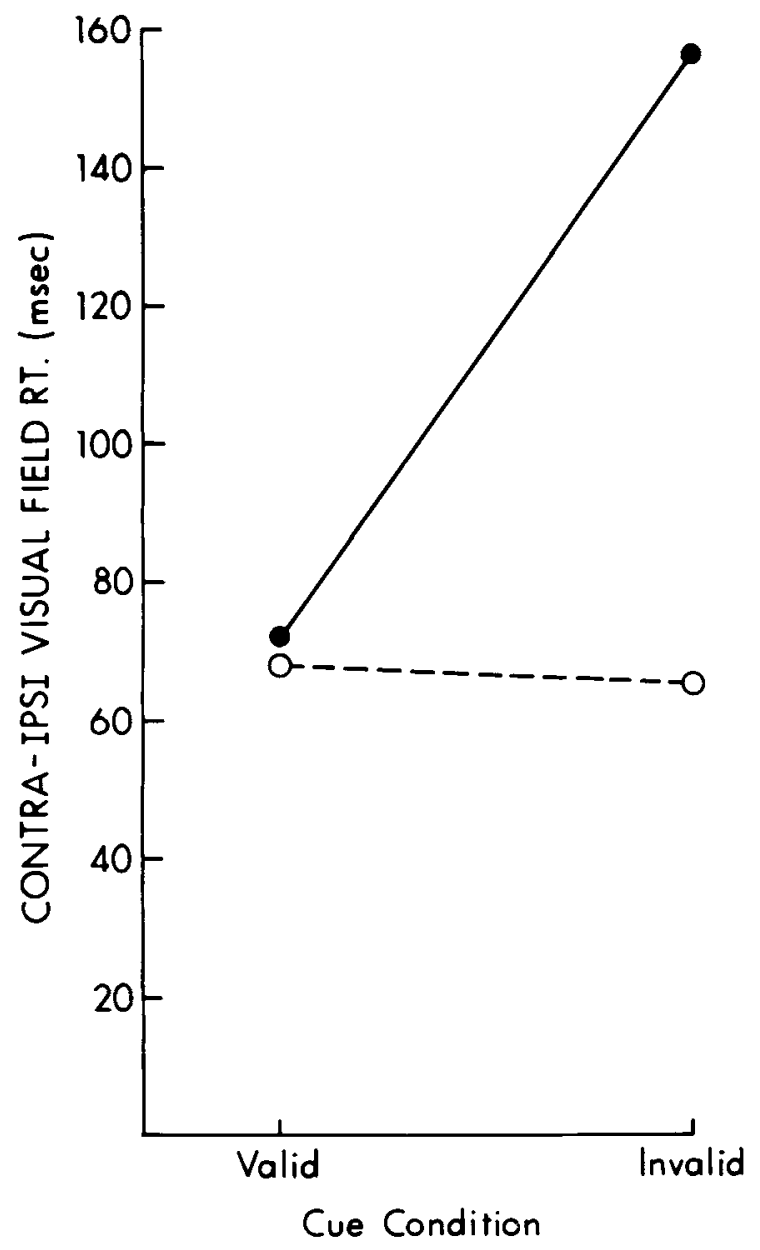

Figure 5. Magnitude of extinction-like reaction time pattern (contralateral minus ipsilateral reaction times) for single (focused; solid line) and dual (divided; dashed line) task blocks of Experiment 1. All data are at 100-msec cue-to-target interval.

no cues are given. We should be able to vary the relationship between valid-cue and no-cue trials, depending on whether they occur in mixed blocks, where a single criterion might be adopted, or in pure blocks, in which different criteria would be allowed.

The second dramatic result of the present experiment was the poor performance of right-sided patients in the no-cue condition. The performance of right-sided patients was clearly worse than that of normals and left-sided patients in the no-cue condition, although they performed virtually identically to the other groups on valid trials. Moreover, even in comparison with their own performance on valid trials, right-sided patients performed poorly in the no-cue condition. Heilman and Van Den Abell (1979) proposed that the right hemisphere is specialized for arousal. They argued that the hypoarousal resulting from damage to the right hemisphere causes patients with right parietal lesions to have more difficulty in spatial tasks than do left parietal patients. A recent theoretical revoew (Tucker \& Williamson, 1984) has also sup- ported the idea that the right hemisphere is more important than the left for arousal.

Our results suggest that left- and right-sided patients have equal problems with disengaging attention to deal with targets, but they raise the possibility that patients with right-sided lesions also have a special problem with maintaining alertness. ${ }^{1}$ This idea is based on the supposition that, since no-cue trials do not provide a warning, the subjects must act to maintain a high level of alertness if they are to sustain fast RTs. If they fail to do so, their performance will suffer in the no-cue condition. If patients with right-sided lesions have difficulty in maintaining their alertness without a warning, their performance would be at a special disadvantage in this condition.

Experiment 2 concerned the degree of separation between the alerting or arousal produced by the cue and the cue's ability to direct attention to the cued location. In many cognitive theories these are seen as separate aspects of attention. The data shown in Table 2 suggest that the omission of a cue selectively increases RTs for patients with right-sided lesions. These patients do not differ from left-sided patients in the ability of the cue to direct attention (invalid-valid RTs). Thus if we are correct in conjecturing that the performance in the no-cue condition is due to normal subjects' adopting a low criterion in pure no-cue blocks, we could then attribute the performance of right-hemisphere lesioned patients to a specific deficit in alertness that appears quite separate from the deficit found in the ability of the cue to direct attention.

\section{EXPERIMENT 2}

To test our conjecture about the nature of the data provided by the no-cue condition, we designed an additional set of experiments with young normal subjects. In Experiment $2 \mathrm{a}$ we tested the idea that the relative speed of responding for blocks of cued trials and for blocks of no-cue trials depended on the subject's level of alertness. We compared blocks of trials in which the time between trials was $500 \mathrm{msec}$ (nearly optimal for alertness) with trials with intervals of 5,000 msec (a suboptimal interval for maintaining alertness). Sanders (1977) showed that long foreperiods produce suboptimal alerting, which has much greater effects for visual than for auditory signals.

In Experiment $2 \mathrm{~b}$ we tested the idea that the advantage of no-cue over valid trials depended on subjects' adopting a low criterion during no-cue blocks. We did this by comparing blocks in which no-cue and cued trials were randomized so that no special criterion could be chosen

Table 2

Mean Reaction Time (in Milliseconds) for No-Cue Blocks Under Focused Task Conditions

\begin{tabular}{|c|c|c|c|c|c|}
\hline \multicolumn{4}{|c|}{ Parietal Patients } & \multicolumn{2}{|c|}{ Normals } \\
\hline \multicolumn{2}{|c|}{ Right } & \multicolumn{2}{|c|}{ Left } & \multirow{2}{*}{$\frac{\text { Old }}{\text { Both }}$} & \multirow{2}{*}{$\frac{\text { Young }}{\text { Both }}$} \\
\hline Contra & Ipsi & Contra & Ipsi & & \\
\hline 820 & 525 & 470 & 430 & 405 & 240 \\
\hline
\end{tabular}


for the no-cue trials with pure blocks in which only nocue or only cued trials were given.

\section{Method}

In Experiment 2a, 12 young normal subjects were run for $2 \mathrm{~h}$. Four pure blocks were used. For two of the blocks the time between trials was $500 \mathrm{msec}$; for the other two, it was $5,000 \mathrm{msec}$. Within each delay condition, one block consisted of no-cue trials in which only a target was presented, and the other block consisted of cued trials ( $80 \%$ valid and $20 \%$ invalid) in which the target followed the cue equally often after 100 and $900 \mathrm{msec}$. Each block had 100 trials.

In Experiment $2 \mathrm{~b}, 10$ young normal subjects were run in a single 1-h session. The experiment was similar to Experiment $2 \mathrm{a}$ except that each subject ran in two mixed blocks of 160 trials. Within each mixed block, there were 96 cued trials (50\% valid) and 64 uncued trials. One mixed block was run with a $500-\mathrm{msec}$ intertrial interval (ITI) (high alertness) and one with a 5,000-msec ITI (low alertness).

\section{Results}

The results of Experiment 2a are shown in Figure 6. The pattern of results in the high-alertness condition was quite similar to that found with normal subjects and leftsided patients in Experiment 1. RTs were fastest in the no-cue condition, intermediate in the valid-cue condition, and slowest in the invalid-cue condition. The low-alertness condition showed a pattern much more like that of the right-sided patients in Experiment 1. The valid trials were slightly faster than the no-cue condition, with the invalidcue trials the slowest.

A statistical analysis of the overall data showed significant effects of ITI $[F(1,11)=6.2, p<.05]$, of validity $[F(2,22)=11.1, p<.01]$, of cue-to-target interval $[F(1,11)=67.1, p<.01]$, of the interaction of validity with cue-to-target interval $[F(1,11)=17.8, p<.01]$, and of the triple-order interaction of ITI and validity with cue-to-target interval $[F(2,22)=3.4, p<.01]$.

The interaction between ITI (alertness) and validity shown in Figure 6 was present at both cue-to-target intervals, but was stronger with the $900-\mathrm{msec}$ interval. This is mainly because the no-cue trials showed a smaller improvement with interval than did the cued trials, since there was no cue to mark the start of the trial. Figure 6 makes it appear that alertness effects were present for both no-cue and invalid trials. In fact, although 11 of the 12 subjects had longer RTs in the low-alertness, no-cue condition than in the high-alertness, no-cue condition, only

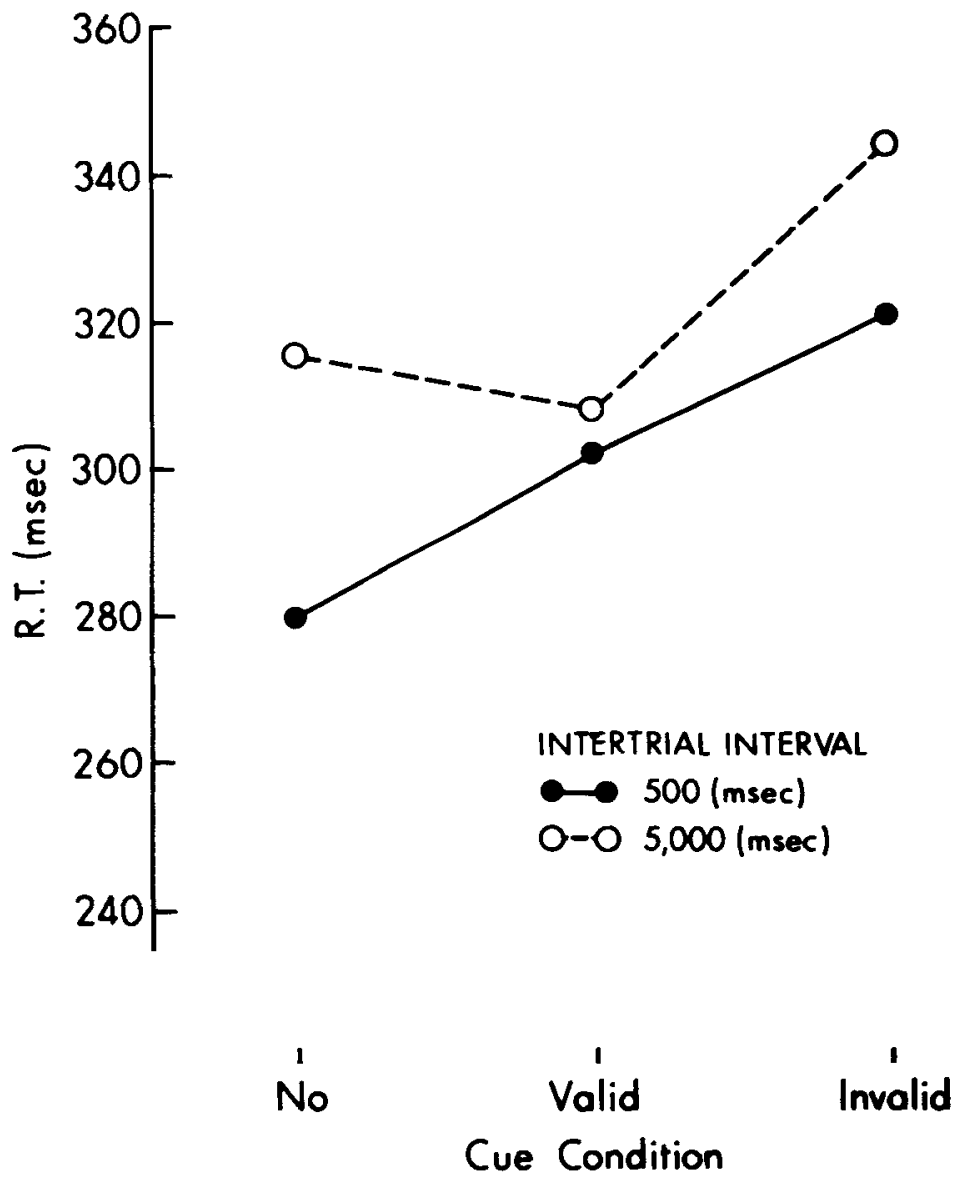

Figure 6. RTs as a function of cue conditions for pure blocks of cued or uncued trials conducted with long $(5,000-\mathrm{msec})$ or optimal $(500-\mathrm{msec})$ intertrial intervals (Experiment 2a). 
7 of the 12 showed an alertness advantage for invalid trials. Thus, subjects appeared to compensate for the suboptimal warning interval quite well in a pure block of cued trials, but not in a pure block of uncued trials.

The results of Experiment $2 b$ are shown in Figure 7. In this experiment, ITI, cue-to-target interval, and cue condition all had significant effects. There was also a cue $X$ interval interaction due to the large improvement in RTs when a cue was present $(p<.01)$. In both alertness conditions, the valid trials had an advantage over the invalid and no-cue trials. This advantage occurred despite the $50 \%$ validity used in the experiment. The no-cue trials had somewhat longer RTs than did the invalid trials, particularly in the low-alertness condition. In comparing the two experiments, it is clear that mixing the block produced a specific disadvantage for the no-cue trials.

\section{Discussion}

These results illustrate the complexity of events that occur even under the relatively simple conditions of Experiment 1. Apparently, the RT to a cued event depends in part on the warning properties the cue provides, in part on the location information provided by the cue, and in part on the inhibition produced by raising the criterion set by subjects in order to resist responding to the cue. By comparing valid with invalid trials in mixed blocks, one can hold the alertness and criterion effects relatively constant to compare the directional effect of the cue.

Experiments $2 \mathrm{a}$ and $2 \mathrm{~b}$ generally confirm our conjecture that the advantage of uncued trials for normal subjects in Experiment 1 results from the subjects' adopting a lower criterion for these blocks. Apparently this is done based on the property of the block and not, or at least not as much, on a trial-by-trial basis. This conclusion follows from our finding that, in mixed blocks, performance was much worse on no-cue trials than on valid trials. The results also suggest that right-sided patients have difficulty in maintaining a high enough level of alertness to perform well when a warning signal is absent. Put another way, patients with right-side lesions fail to lower their criterion for no-cue blocks. Since alertness effects usually result in changes in criterion, these two statements may be equivalent. Our results with normal subjects suggest that a failure to maintain alertness would account for the

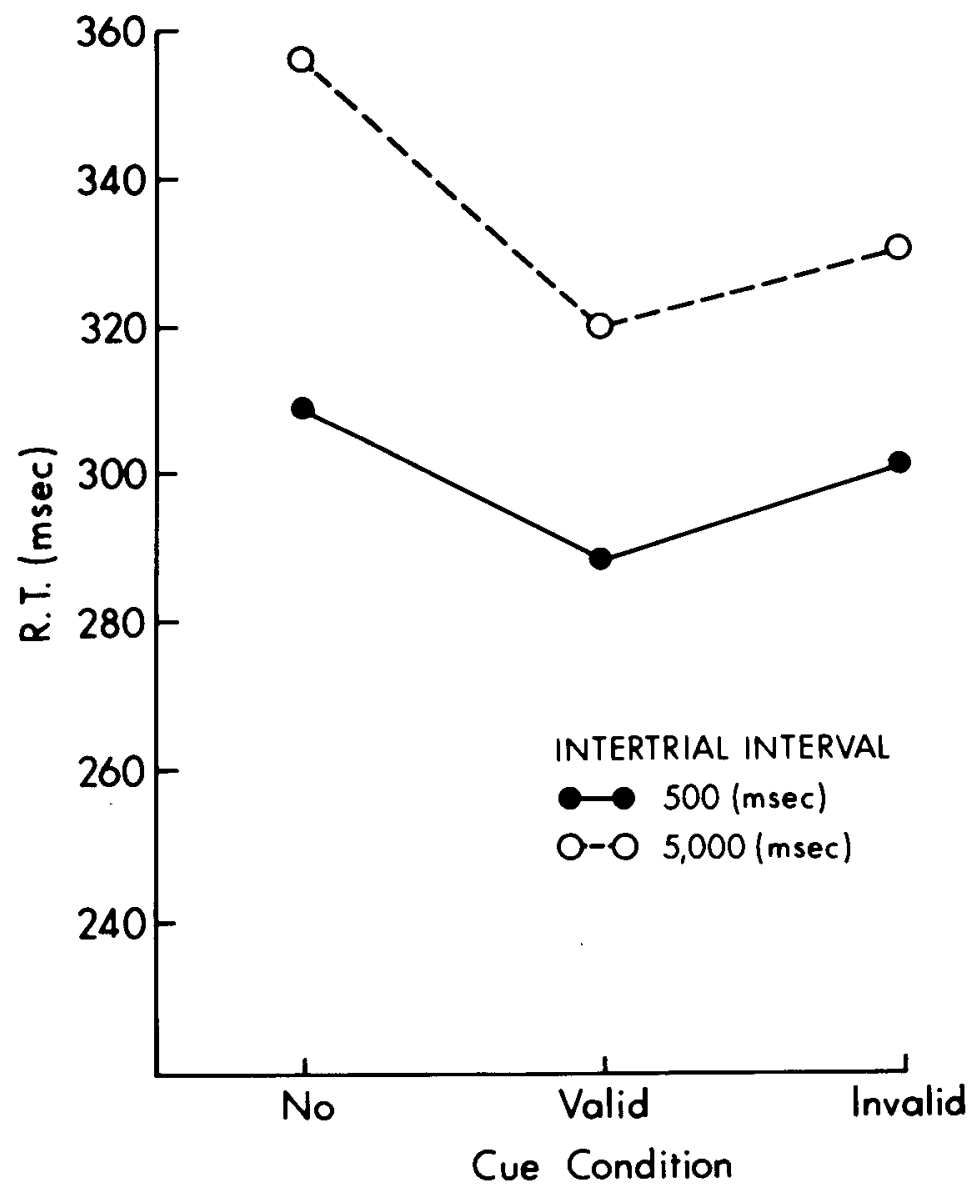

Figure 7. RTs as a function of cue conditions for blocks of mixed cue and nocue trials with long $(5,000-\mathrm{msec})$ and optimal $(500$-msec) intertrial intervals (Experiment $\mathbf{2 b}$ ). 
poor performance of right-sided subjects in the no-cue trials, since their performance resembles that of normals at a lowered level of alerting induced by a suboptimal ITI.

It is also possible to ask whether normals show any differences in alerting when cues are presented directly to the right hemisphere. Heilman and Van Den Abell (1979) suggested that cues delivered to the right hemisphere from the left visual field would result in faster RTs than those found when cues go directly to the left hemisphere. Figure 8 shows RTs from Experiment $2 b$ as a function of which hemisphere first received the target or cue. The lower two curves are for high-alertness conditions; the upper two are for low-alertness conditions. When no cue was provided, the subjects had only the time from the last keypress as the mark of the start of a trial. For cued trials we plotted only valid trials where both cue and target went directly to the same hemisphere. The ANOVA for this breakdown shows that the only significant effects were those of alertness and warning interval. There was a small but nonsignificant trend for performance on the lefthemisphere targets to be better than that on righthemisphere targets under the high-alertness conditions. This trend is in the opposite direction from what would be predicted from a right-hemisphere advantage for alerting. There is no hint that the alerting functions differ for the two hemispheres. Thus, although our patient evidence suggests that right-hemisphere damage reduces the ability to maintain alertness, we are not able to confirm that this effect can be found in normals by varying the location of the warning cue.

\section{CONCLUSIONS}

\section{Parietal Deficit}

The present experiments confirm previous findings concerning the visual-spatial attention system (Baynes, Holtzman, \& Volpe, 1986; Morrow \& Ratcliff, 1987; Posner, Walker, Friedrich, \& Rafal, 1984). When the attention of patients with parietal lesions is summoned to a visual cue, they have a powerful deficit in handling contralateral targets. When attention is at the cued location or when the target is ipsilateral to the lesion, patients show only a small deficit, if any, over the performance of age-related controls. This supports our finding that parietal lesions are specific to the ability to disengage from a stimulus once attention has been committed.

The present study suggests that the right and left parietal lobes are symmetric for the disengage operations, because the advantage of valid over invalid trials is similar for the two groups. Morrow and Ratcliff (1987) used our task and found similar results for right-parietal patients, but little evidence for an overall deficit for left-parietal

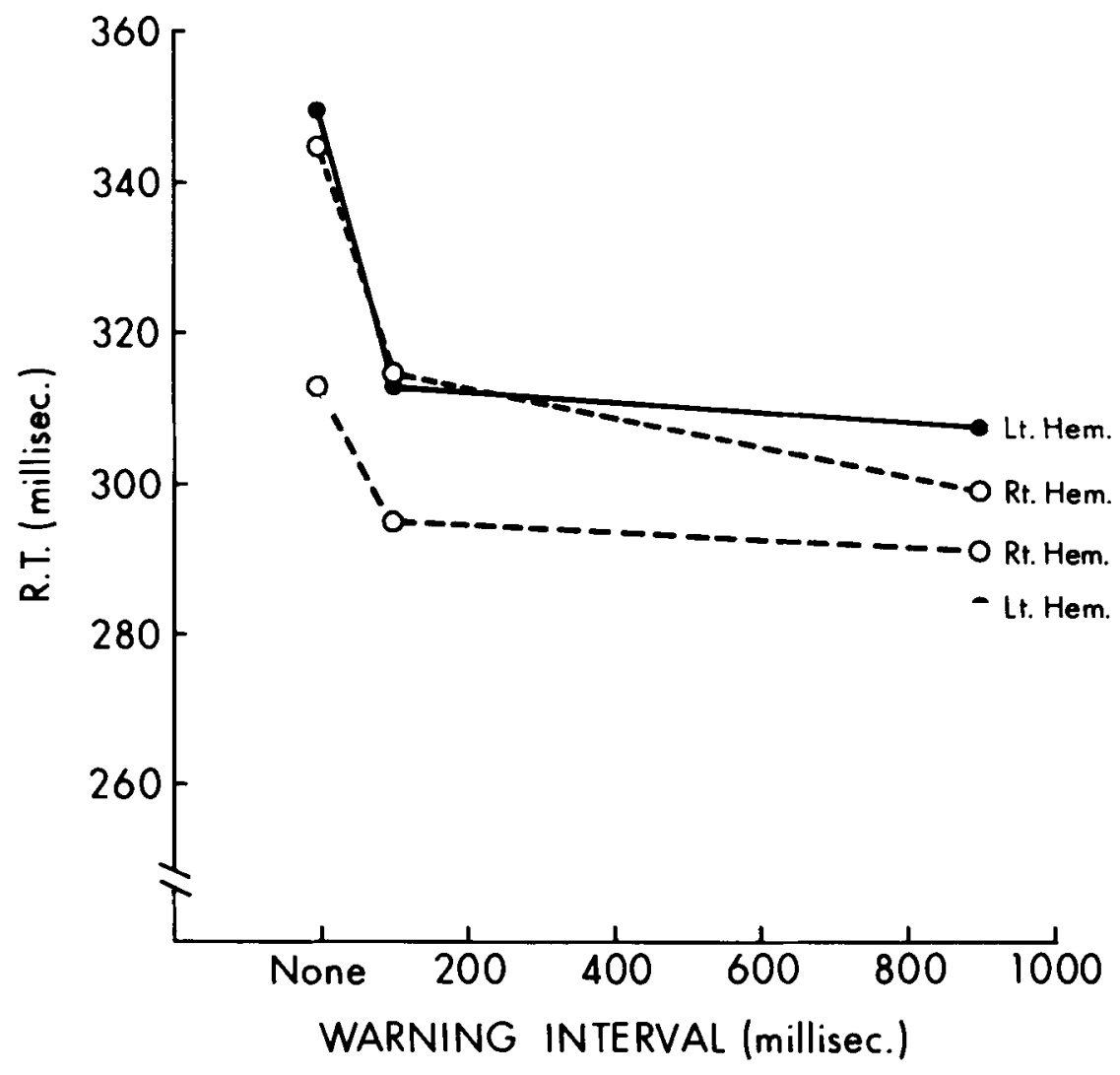

Figure 8. Warning signal function for trials in which the cue or target is presented to the left visual field (right hemisphere) and those for which they are presented to the right visual field (left hemisphere). Data are from no-cue and valid trials of Experiment $2 b$. 
patients. Indeed, in our previous work we also found a weaker effect in left- than in right-parietal patients. ${ }^{2}$ It is well known in clinical neurology that right-parietal patients often show more evidence of neglect than do leftparietal patients (DeRenzi, 1982). However, our data from the no-block trials indicate that the difference between the two groups may lie not in the directionally specific effect of the cut but in its arousal effect (see Alerting, below). Morrow and Ratcliff also found a right-frontal patient who had a disengage deficit. Rafal (1987) presented two frontal patients who showed an orienting deficit, but of a kind different from that found in parietal patients. These results suggest that frontal areas may also play an important role in orienting under some circumstances. It is known that elements of neglect can result from frontal lesions (DeRenzi, 1982). We suggest below that frontal areas may be important in the more general functions of attention common to both spatial orienting and language control.

\section{Is Spatial Attention an Independent Module?}

Visual-spatial attention is one form of selectivity by which information reaches neural systems responsible for conscious report. The parietal damage must involve only a pathway toward conscious report. This is established by the relatively intact performance of parietal patients on valid trials even to targets whose location is contralateral to the lesion. Thus some systems can compensate for the relative inefficiency of the damaged parietal lobe, which argues that higher level attentional systems must be intact.

How does this visual-attention pathway relate to pathways involved in dealing with other aspects of attention? Experiment 1 shows that processing language stimuli (phoneme monitoring or counting backward) delays orienting to the spatial cue. Since the act of orienting requires no overt movement that might interact with the secondary task, it seems reasonable to suppose that attending to nonspatial stimuli interferes directly with the system that shifts visual attention. We know from other work on interference effects (Posner, 1978) that tasks such as counting backward or phoneme monitoring also interfere with most other types of cognitive operations. Moreover, this interference is quite time locked. It is not that the secondary task completely inhibits the attention shift; it simply delays it so that what is usually quite strong at $100 \mathrm{msec}$ is no longer present at that time. In addition, performance on the secondary task suffers from competition from the primary attention-shifting task. These properties suggest that there is a common command system needed both to issue commands that produce spatial orienting and for some aspects of monitoring (e.g., incrementing the count when a target occurs) (Duncan, 1980b).

If one accepts the interference effects found in our patients during the visual-spatial orienting task as evidence for a common attention system, what can we say about this system? Our main finding is that the anatomy of the common system must be different from that of the visual- spatial pathway, because engaging the subject in a language task produces no specific deficit for targets contralateral to the lesion. Since it appears that damage to the parietal lobe manifests itself in a specific deficit in disengaging to deal with contralateral targets, it follows that engaging attention to the language task must not involve the parietal mechanism involved in visual-spatial attention.

In short, our evidence favors two distinct neural systems: a specific visual-spatial system that involves the parietal lobe, and a more general system common to both visual-spatial and language attention. It seems likely that the more general system operates as a command system to allow orienting of visual-spatial or other forms of attention. Since we know that failure of the visual-spatial system means that the patient will be unaware of the target, this second system could be responsible for the specific operations underlying our ability to report the stimulus subjectively.

From previous work in cognitive psychology (Marcel, 1983), it appears that under some conditions a visual stimulus is processed quite deeply, including the production of semantic activation without the subject's being aware of the stimulus. In anatomical terms, this suggests that a good deal of processing by posterior areas of the brain can occur without the subject's being conscious of the event.

It is possible only to speculate on the anatomical basis of the systems common to visual-spatial attention and attention to language. The parietal lobe is closely connected anatomically to areas of the frontal lobe, both on the lateral surface (the dorsolateral prefrontal cortex; Schwartz \& Goldman-Rakic, 1982) and on the medial surface (e.g., the supplementary motor area). In recent studies using positron emission tomography to study regional cerebral blood flow, the supplementary motor area has been found to be active in tasks that involve attention to language (Petersen, Fox, Posner, Mintun, \& Raichle, 1987) and in tasks that involve overt eye movements during visual processing and imagery (Fox, Fox, \& Raichle, 1985; Roland, 1985).

\section{Alerting}

How do the results concerning alerting fit into the operation of selective systems? First, our experiments show that alerting effects are quite independent of the direction of attention. This view arose first from experiments performed many years ago in which primes and warning signals were shown to have additive effects on improvement in RTs (Posner \& Boies, 1971). At that time it was pointed out that the source of alerting effects was likely to be subcortical arousal systems, since EEG evidence of alerting was found in both hemispheres of split-brain monkeys even when the signal went only to one hemisphere (Gazzaniga \& Hillyard, 1973). These subcortical systems seem to influence stimulus processing by acting on a higher level attention system rather than on input pathways (Posner, 1975). This argument was based on evidence favoring a criterion shift because increased error 
rates usually accompany faster RTs following warning signals.

The present data extend this view by showing that patients with right-side lesions have special difficulty in maintaining a high level of alertness during a brief delay between trials. This difficulty does not affect their ability to use warning signals or their ability to shift attention in the cued direction.

According to our earlier argument (Posner, 1978), deficits in alerting affect higher level attention systems, rather than the activation of pathways by which information is accumulated. In terms of our present argument, they would affect the frontal attention-command system rather than, or more strongly than, the posterior visual-spatial attention system. In consequence, deficits in alerting would retard the commands needed to activate the posterior system. Such poorly maintained alerting in the absence of specific cues might explain why right-parietal patients show a deficit in performance in natural and clinical situations. In accord with this possibility, recent evidence suggests that the right hemisphere may be more closely involved in the arousal of cortex by norepinephrine and serotonin than is the left hemisphere (Tucker \& Williamson, 1984).

\section{Hierarchical Distributed Network}

Figure 9 illustrates the view of covert orienting under dual-task conditions that appears to be consistent with our data. The top of the figure shows the task events. Each rectangle in the lower part of the figure contains the mental operations involved in the performance of the task. The last four operations have been discussed in some detail in our previous work on visual orienting (Posner, Walker, Friedrich, \& Rafal, 1984). When a cue arrives, the subject's attention is disengaged from its current location. We have shown that damage to the posterior parietal lobe selectively interferes with this operation, which suggests that the parietal lobe is a necessary structure for performing it. Attention is then moved to the location of the cue. We have shown that the speed of moving attention to a cued location is slowed by damage to the midbrain (Posner et al., 1985). We assume, then, that attention must engage the location of the target to generate the arbitrary detection response required by the task (Posner, 1980). When attention is drawn to a location by a cue and is subsequently withdrawn, there is a long-lasting inhibitory effect at the cued location, which we have called inhibition of return (Maylor, 1985; Posner \& Cohen, 1984).

This paper concerns the three mental operations indicated at the top of Figure 9, namely, alert, interrupt, and localize. The first, we assume, is a general effect of the cue. It is well known that any event that can be used as a warning signal produces a negative shift in the EEG (Donchin, 1984). This negative shift, called the contingent negative variation (CNV), is the brain sign most closely associated with a general alerting function. It is thought to involve the reticular activation system and to operate first on prefrontal sites and then move backward over the scalp, the exact details depending on the task to be performed. The frontal distribution to the CNV makes this sign attractive as the basis for interrupting the ongoing language processing in which our subjects are engaged in the dual-task conditions. On the basis of the close anatomical connections of prefrontal cortex to both parietal and language areas and to the cortical origin of the CNV, we speculate that prefrontal cortex is the common site of interference between the language and spatial attention tasks. Because the subjects engaged in the language task showed retarded orienting toward the cue, we believe that an interruption of the ongoing language task must be a necessary condition to produce the four operations listed at the bottom of the figure. In this sense, the system involved in the interrupt operation is in command of the posterior systems involved in the final four operations.

The localize operation is necessary for the coordinates of the cue to direct the covert shift of attention. A model for this operation was described by Koch and Ullman (1985), who assumed that it involves areas of primary visual cortex that must also be directly activated by the

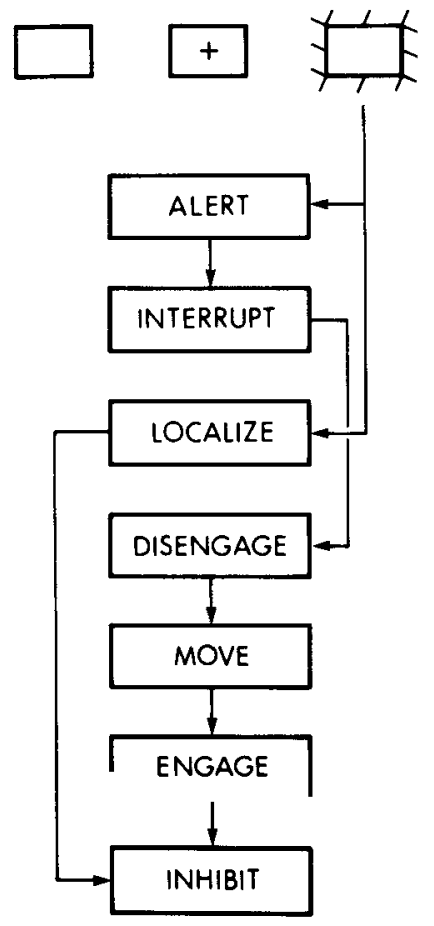

Figure 9. An overview of the hierarchy of attention involved in the covert orienting task described in this paper. The presentation of the cue produces a change in alertness thought to operate via reticular activating system on frontal sites. Alerting can be used to interrupt the current attended activity, which, in our dual-task conditions, is attending to phoneme monitoring. The cue also provides information about its location. Once ongoing language processing is interrupted, the posterior parietal lobe acts to disengage attention from its current focus and move it to the location of the cue. The cue is also known to set up an inhibition of return that leads to slower performance to events at the cued location once attention has been withdrawn (Posner, Choate, Rafal, \& Vaughan, 1985). 
cue. Koch and Ullman also described how the process of localizing the cue might lead naturally to the inhibition of return.

Mesulam (1981) attempted to distinguish among several views of the way in which brain systems function to control spatial attention. He called these general views center theories, network theories, and holistic theories. The center theory regards spatial attention as the single system. The network theory views components of the function as assigned to quite distinct neural systems. The holistic theory regards attention as a general property of the brain. The data reviewed by Mesulam favor a network theory. Our data also support a network approach. The anatomical separation between the visual-spatial attention system and the higher level common system argues against a single center, and the degree of anatomical specificity found for visual-spatial attention argues against any holistic view. However, our findings suggest two additions to the network view.

First, we try to specify the components of covert orienting of spatial attention (see Figure 9). In this sense, our viewpoint is cognitive as well as anatomical. This is quite similar to what has been done by Kosslyn (1980) for visual imaging. Indeed, some of the operations in covert visual attention may be the same as those used in scanning visual images. It is also in the spirit of recent work in imagery to attempt to relate the mental operations posited by the model to the neural systems that support them (Farah, 1984). In this sense our approach is also anatomical.

Our results favor a second modification of Mesulam's network theory. This modification might be called a hierarchical network. We find that some neural systems related to attention seem to coordinate or to control the action of other systems. Thus within the visual-spatial system, the parietal mechanism must act to disengage attention prior to its being moved to the target. The midbrain centers shown to affect the move operation would have to be controlled by the operation of the cortical centers responsible for the disengage operation. Similarly, it appears that the posterior areas responsible for spatial orienting as a whole are controlled by a higher level frontal system.

We believe that the hierarchical network viewpoint is very much in accord with the general spirit of findings in both neurophysiology and cognitive psychology. Neurophysiology often views the operation of higher centers as acting to tonically inhibit lower systems and, through feed-forward mechanisms, to produce phasic potentiation of activity (Mountcastle, 1978). In cognitive psychology, central-attention theories offer a necessary means of coordination among a number of semiindependent codes that are activated by input (Keele \& Neil, 1978; Posner, 1978; Treisman \& Gelade, 1980).

There are selection mechanisms within each sensory system (Hillyard \& Kutas, 1983; Näätänen, 1982) that serve to gate some information and to potentiate other information sources. At the level of the cortex, informa- tion from different sensory systems (e.g., vision and audition) must be integrated when it relates to the same cognitive system (e.g., spatial attention, object identification, language). Indeed, Posner and Henik (1983) compared the effectiveness of stimuli in producing mutual interference and facilitation when both were within the same modality (e.g., vision) but in different cognitive systems (e.g., spatial vs. language), and when they were within the same cognitive system (e.g., language) but in different modalities (e.g., vision vs. audition). Our results show that, at least under the conditions of our test, stimuli within the same cognitive system produce more mutual interaction than those that share only input modality. This point argues for a level of selection that integrates separate sensory systems.

It seems reasonable to suppose that stimuli in different cognitive systems (e.g., language and spatial location) must be coordinated at some level. The results of the present study show that the principle of distributed but hierarchical networks can be applied to this problem. Although the disengage operation can be specific to a mechanism within a cognitive system (e.g., visual-spatial attention), it also appears that more general systems are required to permit selection when more than one cognitive system is involved. We find that when a subject is engaged in a language operation, there is a clear reduction in efficiency of spatial orienting. The hierarchical network idea allows us to see why damage to a particular location in the central nervous system produces a deficit in operations specific to one cognitive system (e.g., spatial orienting or language), whereas damage to other locations may produce more widespread attentional deficits that are not specific to any cognitive system.

\section{REFERENCES}

Bashinski, H. S., \& Bachrach, V. R. (1980). Enhancement of perceptual sensitivity as the result of selectively attending to spatial locations. Perception \& Psychophysics, 28, 241-248.

Baynes, K., Holtzman, J. D., \& Volpe, B. T. (1986). Components of visual attention: Alterations in response pattern to visual stimuli following parietal lobe infarction. Brain, 109, 99-114.

DERENZI, E. (1982). Disorders of space exploration and cognition. New York: Wiley.

Donchin, E. (1984). Report of Panel III: Preparatory Processes. In E. Donchin (Ed.), Cognitive psychophysiology (pp. 179-219). Hillsdale, NJ: Eribaum.

Downing, C. J., \& Pinker, S. (1985). The spatial structure of visual attention. In M. I. Posner \& O. S. M. Marin (Eds.), Attention and performance $X I$ (pp. 171-188). Hillsdale, NJ: Erlbaum.

DunCAN, J. (1980a). The demonstration of capacity limitation. Cognitive Psychology, 12, 75-96.

Duncan, J. (1980b). The locus of interference in the perception of simultaneous stimuli. Psychological Review, 87, 272-300.

FARAH, M. J. (1984). The neurological basis of mental imagery: A componential analysis. Cognition, 18, 245-272.

Fox, P. T., Fox, J. M., \& RaIchle, M. E. (1985). The role of cerebral cortex in the generation of voluntary saccades: A positron-emission tomographic study. Journal of Neurophysiology, 54, 348-369.

Gazzaniga, M., Hillyard, S. A. (1973). Attention mechanisms following brain bisection. In S. Kornblum (Ed.), Attention and performance IV (pp. 221-237). New York: Academic Press. 
Heilman, K. M., \& Van Den Abell, T. (1979). Right hemisphere dominance for mediating cerebral activation. Neuropsychologia, 17, 315-321.

Hillyard, H. C., \& KuTAs, M. (1983). Electrophysiology of cognitive processes. Annual Review of Psychology, 34, 33-61.

Hughes, H. C., \& Zimba, L. D. (1985). Spatial maps of directed visual attention. Journal of Experimental Psychology: Human Perception \& Performance, 11, 409-430.

Jonides, J. P. (1981). Voluntary versus automatic control over the mind's eye. In J. Long \& A. Baddeley (Eds.), Attention and performance $I X$ (pp. 187-204). Hillsdale, NJ: Erlbaum.

KeELE, S. W., \& NeIL, W. T. (1978). Mechanisms of attention. In E. C. Carterette (Ed.), Handbook of perception (Vol. 9, pp. 3-47). New York: Academic Press.

KerR, B. (1973). Processing demands during mental operations. Memory \& Cognition, 1, 401-412.

Koch, C., \& Ullman, S. (1985). Selective visual attention: Towards the underlying neural circuitry. Human Neurobiology, 4, 219-227.

KossLYN, S. W. (1980). Image and mind. Cambridge, MA: Harvard University Press.

LABERGE, D. L. (1973). Identification of two components of the time to switch attention. In S. Kornblum (Ed.), Attention and performance $I V$ (pp. 71-85). New York: Academic Press.

Lansman, M, FarR, S., \& HunT, E. (1984). Expectancy and dual task of interference. Journal of Experimental Psychology: Human Perception \& Performance, 10, 195-204.

MarCel, A. J. (1983). Conscious and unconscious perception: Experiments on visual masking and word recognition. Cognitive Psychology, 15, 197-237.

MAYLOR, E. A. (1985). Facilitatory and inhibitory components of orienting in visual space. In M. I. Posner \& O. S. M. Marin (Eds.), Attention and performance $X I$ (pp. 189-289). Hillsdale, NJ: Erlbaum.

Mesulam, M. M. (1981). A cortical network for directed attention and unilateral neglect. Annals of Neurology, 10, 309-325.

Morrow, L. A., \& RatclifF, G. G. (1987). Attentional mechanisms of clinical neglect. Journal of Clinical \& Experimental Neuropsychology, 9, 74-75.

MountCASTLE, V. B. (1978). Brain mechanisms for directed attention. Journal of the Royal Society of Medicine, 71, 14-27.

NÄ̈TÄNA, R. (1982). Processing negativity: An event related potential reflection of selective attention. Psychological Bulletin, 92, 605-640.

Petersen, S. E., Fox, P. T., Posner, M. I., Mintun, M. A., \& RAICHLE, M. E. (1987). Focal brain activity during visual language tasks are measured with averaged PET images of evoked CBF change. Society for Neuroscience Abstracts, 12, 1261.

Pinker, S. (1980). Mental imagery and the third dimension. Journal of Experimental Psychology: General, 109, 354-371.

PosNer, M. I. (1975). Psychobiology of attention. In M. Gazzaniga \& C. Blakemore (Eds.), Handbook of psychobiology (pp. 441-480). New York: Academic Press.

Posner, M. I. (1978). Chronometric explorations of mind. Hillsdale, NJ: Erlbaum.

PosNer, M. I. (1980). Orienting of attention. Quarterly Journal of Experimental Psychology, 32, 3-25.

Posner, M. I., \& BoIEs, S. J. (1971). Components of attention. Psychological Review, 78, 391-408.

Posner, M. I., Choate, L., Rafal, R. D., \& Vaughan, J. (1985). Inhibition of return: Neural mechanisms and function. Cognitive Neuropsychology, 2, 211-228.

Posner, M. I., \& Cohen, Y. (1984). Components of visual orienting. In H. Bouma \& D. G. Bowhuis (Eds.), Attention and performance $X$ (pp. 531-556). Hillsdale, NJ: Erlbaum.

Posner, M. I., Cohen, Y., Choate, L., Hockey, R., \& Maylor, E. (1984). Sustained concentration: Passive filtering or active orienting. In S. Kornblum \& J. Requin (Eds.), Preparatory states and processes (pp.49-65). Hillsdale, NJ: Erlbaum.

Posner, M. I., Cohen, Y., \& Rafal, R. D. (1982). Neural systems control of spatial orienting. Proceedings of the Royal Society of London $B, 298,187-198$.
Posner, M. I., \& HeNIK, A. (1983). Isolating representational systems. In J. Beck, B. Hope, \& A. Rosenfeld (Eds.), Human and machine vision (pp. 395-412). New York: Academic Press.

Posner, M. I., Walker, J. A., Friedrich, F. J., \& Rafal, R. D. (1984). Effects of parietal lobe injury on covert orienting of visual attention. Journal of Neuroscience, 4, 1863-1874.

RaFAL, R. D. (1987). Frontal lobe lesions slow the movement of visual attention. Neurology, 37(Suppl. 1), 128.

Remington, R., \& PIERCe, L. (1984). Moving attention and evidence for time-invariant shifts of visual selective attention. Perception \& Psychophysics, 35, 393-399.

Roland, P. E. (1985). Cortical organization of voluntary behavior in man. Human Neurobiology, 4, 155-167.

SANDERS, A. (1977). Structural and functional aspects of the reaction process. In S. Dornic (Ed.), Attention and performance VI (pp. 325). Hillsdale, NJ: Erlbaum.

Schwartz, M. L., \& Goldman-Rakic, P. S. (1982). Single cortical neurons have axon colaterals to ipsilateral and contralateral cortex in fetal and adult primates. Nature, 299, 154-156.

SHaw, M. L. (1984). Division of attention among spatial locations. In H. Bouma \& D. G. Bouwhuis (Eds.), Attention and performance $X$ (pp. 109-124). Hillsdale, NJ: Erlbaum.

SHEPARD, R. N. (1978). The mental image. American Psychologist, 33, 125-137.

Shulman, G. L., Remington, R., \& Mclean, J. P. (1979). Moving attention through visual space. Journal of Experimental Psychology: Human Perception \& Performance, 5, 522-526.

Treisman, A. M., \& Gelade, P. A. (1980). A feature integration theory of attention. Cognitive Psychology, 12, 97-136.

Tsal, Y. (1983). Movements of attention across the visual field. Journal of Experimental Psychology: Human Perception \& Performance, 9, 523-530.

Tucker, P. M., \& Williamson, P. A. (1984). Asymmetric neural control in human self-regulation. Psychological Review, 91, 185-215. Ullman, S. (1984). Visual routines. Cognition, 18, 97-159.

Wurtz, R. H., Goldberg, M. E., \& Robinson, D. L. (1980). Behavioral modulation of visual responses in monkey. Progress in Psychobiology \& Physiological Psychology, 9, 42-83.

\section{NOTES}

1. Criterion bias has been widely discussed in signal detection theories of attention (Shaw, 1984). In our view, attention might work through a criterion shift or through a change in $d^{\prime}$. Indeed, in a multilevel system a bias at one level may be a sensitivity change at some other level. Alertness seems to involve a criterion shift in that it does not affect the rate of information gained from a cue, but only the response of some other system. It is the goal of this study to find out something about the nature of that other system. If patients with lesions of the right hemisphere have a problem in adopting a lowered criterion in no-cue blocks, that would be one sort of constraint on the system involved in establishing the criterion. Elsewhere (Posner \& Cohen, 1984) we have discussed other constraints on the kind of criteria that may relate to attention shifts.

2. One has to be cautious about comparisons between small groups of left- and right-lesioned patients. The similarity of the RTs for valid and invalid trials in Figure 1 is quite striking. Moreover, in a previously reported study (Posner, Walker, Friedrich, \& Rafal, 1984) we found no significant differences between left- and right-lesioned patients even when we tried to equate lesion size. However, we cannot dismiss the possibility that differences in lesions mask a difference between leftand right-lesioned patients. However, the massive difference between groups in the no-cue conditions suggests that whatever is being measured in that condition is a far more important difference than that found for the attention shift as measured by the difference between valid and invalid trials.

(Manuscript received February 11, 1987; revision accepted for publication April 19, 1987.) 